\title{
Subdiagnóstico de delirium en adultos mayores hospitalizados
}

\author{
Marcela Carrasco G ${ }^{1,2}$, Trinidad Hoyl $M^{1,2}$, \\ Pedro Paulo Marín $L^{1,2}$, Jaime Hidalgo $A^{a}$, \\ Carmen Lagos $D^{b}$, Cristóbal Longton $B^{b}$, Pamela Chávez $B^{c}$, \\ Eduardo Valenzuela $A^{1,2}$, D omingo Arriagada $M^{2}$. \\ Delirium in Chilean elderly \\ inpatients: an overlooked problem
}

Background: Delirium is a common underdiagnosed and undertreated problem in elderly inpatients, associated to higher morbidity, mortality and health cost. Aim: To evaluate the prevalence of delirium at hospital admission in medically ill elderly patients and the attending physician's diagnosis and treatment of delirium. Patients and methods: In a prospective and descriptive study, consecutive patients aged 65 years or more, admitted to an internal medicine ward were evaluated by independent physicians, during the first $48 \mathrm{~h}$ of admission, to asses the presence of delirium. Diagnosis of delirium was based on the Confusion Assessment Method. Medical and nurse records were reviewed. Family was interviewed when necessary. Results: One hundred eight patients ( $52 \%$ women, age range $65-94$ years) with an APACHE II score of $11.6 \pm 5$, were evaluated. Fifty seven patients (53\%) had delirium (32\% hyperactive, $72 \%$ hypoactive and $5 \%$ mixed). Delirium prevalence was significantly higher in older patients (66\% among those aged 75 years or older versus $30 \%$ in younger, $\mathrm{p} \varangle 0.05$ ) and among patients with more severe conditions ( $88 \%$ among those with an APACHE score over 16 versus $47 \%$ below that value, $p \varangle 0.05$ ). Medical records of patients with delirium showed that this diagnosis was present only in $32 \%$ and cognitive deficit was described in $73 \%$. Ten percent of patients with delirium received sedative medication and $38 \%$ were physically restricted. There were no environmental interventions to prevent or control delirium. Conclusions: Delirium in elderly inpatients at this unit is an extraordinarily prevalent problem, seriously under diagnosed (68\%) and under treated. This study should alerts our medical community to improve the diagnosis and management of delirium in elderly inpatients (Rev Méd Chile 2005; 133: 1449-54).

(Key Words: Aged; Delirium; Frailelderly; Hospitalized)

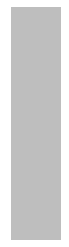

\footnotetext{
Recibido el 9 de agosto, 2004. Aceptado el 4 de julio, 2005.

${ }^{1}$ Programa de Geriatría, ${ }^{2}$ Departamento de Medicina Interna, Pontificia Universidad Católica de Chile.

a Residente del Programa de Medicina Interna, Departamento de Medicina Interna, Pontificia Universidad Católica de Chile.

${ }^{b}$ Alumno de Medicina, Pontificia Universidad Católica de Chile.

${ }^{\mathrm{c}}$ Residente del Programa de Geriatría.
}

Correspondencia a: Dra. Marcela Carrasco G. Programa de Geriatría, Departamento de Medicina Interna, Facultad de Medicina, Pontificia Universidad Católica de Chile. Casilla 114 D, Santiago de Chile. Fax: 354-6985. Fono: 3543030. E mail: mcarras@med.puc.cl 
$\mathrm{E}^{1}$ delirium es un síndrome neuropsiquiátrico de inicio agudo y curso fluctuante, caracterizado por compromiso de conciencia, atención y pensamiento desorganizado, cuyo origen es muchas veces multifactorial ${ }^{1-3}$. La prevalencia en pacientes hospitalizados fluctúa entre 10\% y $30 \% 4$, y en el grupo más vulnerable, que son los pacientes mayores de 65 años (AM) hospitalizados, puede alcanzar hasta $60 \% 1,5-7$.

El delirium en AM hospitalizados se asocia a una evolución desfavorable, los pacientes que lo presentan tienen mayores complicaciones y mortalidad intrahospitalaria, mayor deterioro funcional, prolongación de la estadía hospitalaria y después del alta, mayor institucionalización en unidades de larga estadía y mayor riesgo de morir en los meses que siguen a la hospitalización ${ }^{1,2,4}$. Lo anterior, conduce a un importante aumento en los costos de atención de salud comparados con los AM que no lo presentan ${ }^{1-8}$.

A pesar de su importancia, tradicionalmente el delirium ha sido subvalorado y no ha sido reconocido como una entidad que requiere un diagnóstico y manejo específico, independiente de la causa que lo origina ${ }^{3}$. El subdiagnóstico alcanza cifras entre $32 \%$ y $67 \% 1,3,9$, hecho que no es menor, ya que hay evidencia que eso se asocia con peor pronóstico ${ }^{3,10}$, y se pierden posibilidades de usar distintas estrategias que podrían modificar su aparición y optimizar su manejo ${ }^{11-14}$.

En Chile, la población de AM está creciendo y ya es uno de los principales usuarios de los servicios hospitalarios, fundamentalmente los públicos, que acogen a más del $80 \%$ de ellos ${ }^{15}$. Existen dos estudios chilenos publicados que abordan el tema del delirium. Uno estudió 406 pacientes de 11 a 90 años de un servicio de medicina general y mostró una prevalencia de delirium de $3 \%{ }^{16}$, el otro evaluó 64 pacientes de 19 a 90 años, de una unidad de medicina de cuidados intermedios y mostró una prevalencia de delirium de $41 \%^{17}$. No existen estudios chilenos sobre la magnitud del problema específicamente en el grupo más vulnerable, los AM, y menos aún se conoce el nivel de diagnóstico ni el tipo de manejo que reciben estos pacientes en hospitales públicos.

El objetivo del presente estudio fue conocer la incidencia de delirium en adultos mayores, durante las primeras $48 \mathrm{~h}$ de hospitalización en un Servicio de Medicina de un hospital público, la frecuencia de formulación del diagnóstico de delirium y el manejo del cuadro, por parte de los médicos tratantes. Además, comparar la mortalidad y estadías hospitalarias entre los pacientes que desarrollaron delirium y los que no.

\section{PACIENTES Y MÉTOdO}

Se diseñó un estudio prospectivo y observacional, en el que los sujetos de estudio fueron todos los pacientes con 65 años o más, que ingresaron al Servicio de Medicina del Hospital de Urgencia de la Asistencia Pública, desde su domicilio, entre el 3 y el 28 de agosto de 2003.

Se excluyó a quienes no pudieron ser evaluados en las primeras $48 \mathrm{~h}$ de su ingreso, aquellos ingresados con diagnóstico de accidente cerebro vascular agudo, con intubación endotraqueal o en coma. También, los pacientes en quienes no fue posible establecer con claridad el estado cognitivo basal (con alta sospecha de demencia de base y sin información de familiares).

Los pacientes fueron evaluados durante las primeras $48 \mathrm{~h}$ de su ingreso, por residentes de Geriatría capacitados e independientes del equipo tratante. Los pacientes se clasificaron según la presencia 0 ausencia de delirium mediante entrevista con el paciente, utilizando pruebas de orientación, atención y memoria. Se utilizó los criterios diagnósticos de delirium del «onfusion Assessment Method CAM ${ }^{\star 8}$. En aquellos casos en que existió duda del nivel cognitivo basal del paciente se entrevistó a la familia o cuidador.

Se revisó la ficha médica, al ingreso a la unidad, consignando datos demográficos, años de educación, la presencia de examen mental, descripción del estado de conciencia y la presencia del diagnóstico explícito de «elirium»o «Síndrome Confusional Agudo»y el diagnóstico principal de ingreso.

Se registró las medidas de manejo indicadas, por parte del equipo tratante, tanto ambientales (reorientación, iluminación, restricción física, movilización, etc.) como farmacológicas, consignando el tipo de medicamento y la dosis administrada.

Se revisó la ficha de enfermería registrando la descripción del estado mental del paciente, la presencia de agitación psicomotora y las medidas implementadas para su manejo.

El diagnóstico principal de ingreso, se agrupó en causas cardiovasculares (cardiopatía coronaria, 
insuficiencia cardíaca, etc.), infecciosas (que incluyó infección urinaria, neumonía, etc.), pulmonares (EPOC descompensadas, asma, etc.), metabólicas (diabetes mellitus descompensada, insuficiencia renal o hepática, etc.), digestivas (hemorragias digestivas, dolor abdominal, etc.) y otros. Para medir el nivel de gravedad de la enfermedad médica del paciente se calculó el índice APACHE II ${ }^{19}$.

Los pacientes con delirium fueron clasificados en delirium hiperactivos, hipoactivos 0 mixtos $^{20}$. Para lo cual se consideró la entrevista clínica y la descripción en la ficha médica o de enfermería.

El grupo de pacientes sin delirium en las primeras $48 \mathrm{~h}$ de su ingreso, fue seguido diariamente mediante entrevista utilizando el CAM y revisión de las fichas médicas y de enfermería para detectar la aparición de delirium durante su hospitalización.

Se revisó las epicrisis de los pacientes estudiados consignando la duración de la hospitalización y su condición y destino al alta (vivo a domicilio, vivo trasladado a otro centro hospitalario o fallecido).

A todos los pacientes se les solicitó su consentimiento informado verbal, previo a la evaluación, para participar en el estudio. Por razones éticas, cada vez que se detectó un paciente con delirium, se le informó al médico tratante.

Estadística. Los resultados fueron analizados usando el programa estadístico SPSS, se utilizó el test Chi cuadrado para variable categónica, test de t de Student para comparar medias, prueba de comparación de proporciones para porcentajes, se consideró estadísti- camente significativo un $\mathrm{p} \varangle 0,05$ y se calculó Odds ratio (OR) con intervalos de confianza (IC) de 95\%.

\section{RESULTADOS}

Fueron evaluados en este estudio, cumpliendo con los criterios de inclusión, un total de 108 pacientes, siendo $51,9 \%$ mujeres $(n=56)$, con una edad promedio de 78 años (65-94 años), educación promedio 5,0 años (0-15 años). El 76\% de los pacientes fue entrevistado el día de su hospitalización y $24 \%$ al día siguiente. El APACHE II promedio del grupo total fue de 11,6 puntos (DS 5,0).

Los sujetos excluidos, según los criterios definidos en forma previa al inicio del estudio, fueron 59 pacientes, detallados a continuación: traslados a otros centros hospitalarios o fallecimiento antes de $24 \mathrm{~h}(\mathrm{n}=5)$, incapacidad de comunicarse verbalmente para ser evaluados por intubación endotraqueal 0 coma $(n=3)$, imposibilidad de conocer estado cognitivo basal $(\mathrm{n}=2)$, accidente cerebrovascular agudo $(n=33)$ y pacientes ingresados al servicio de medicina, trasladados desde otras unidades del hospital, con más de $48 \mathrm{~h}$ de hospitalización $(\mathrm{n}=16)$.

Pacientes con delirium al ingreso. Un total de 57 de los 108 pacientes estudiados cumplió criterios de delirium al ingreso (52,8\%). Las características generales de los grupos se describen en la Tabla 1. Destaca que el grupo de pacientes con delirium al

Tabla 1. C aracterísticas generales de los adultos mayores evaluados en el Servicio de M edicina del H ospital de U rgencia de la Asistencia Pública, agosto 2003

\begin{tabular}{|lccrr|}
\hline Características & $\begin{array}{c}\text { Grupo total } \\
\mathrm{N}=108\end{array}$ & $\begin{array}{c}\text { Con Delirium } \\
\mathrm{N}=57\end{array}$ & $\begin{array}{c}\text { Sin Delirium } \\
\mathrm{N}=51\end{array}$ & $\mathrm{p}$ \\
\hline Edad (años prom) & 78,1 & 80,5 & 75,3 & $<0,05$ \\
Sexo (\% mujeres) & 51,9 & 57,9 & 45 & $\mathrm{NS}$ \\
Educación (años prom) & 5,0 & 3,5 & 6,1 & $<0,05$ \\
APACHE II (puntaje prom) & 11,6 & 13,2 & 9,6 & $<0,05$ \\
Estadía hospitalaria (días prom) & 8,1 & 10,2 & 5,8 & $<0,05$ \\
Diagnóstico Principal (\%) & & & & \\
* Cardiovascular & 28,7 & 14,0 & 45,1 & $<0,05$ \\
* Infeccioso & 38 & 50,9 & 23,5 & $<0,05$ \\
* Digestivo & 13,9 & 7 & 21,6 & NS \\
* Metabólico & 8,3 & 15,8 & 0 & $<0,05$ \\
* Otros & 11,1 & 12,3 & 9,8 & NS \\
\hline
\end{tabular}


ingreso, en comparación con los que ingresaron sin delirium $(n=51)$, fue significativamente de mayor edad (66,2\% de los mayores de 75 años versus el $30 \%$ de los menores de 75 años presentó delirium al ingreso $(p<0,05)$ o visto como OR, el delirium fue 4,5 veces más frecuente en los mayores de 75 años (IC 1,96-10,60). Además, quienes presentaron delirium en las primeras $48 \mathrm{~h}$ tenían un mayor índice de gravedad de enfermedad, el OR de presentar delirium teniendo un APACHE II mayor o igual a 16 fue de 4,81 (IC 1,26-18,29). Comparando otras características destaca que tenían significativamente menos años de educación y no hubo diferencias significativas en el género de los pacientes entre ambos grupos.

Según el tipo de delirium, se distribuyeron de la siguiente forma: $22,6 \%$ hiperactivo, $71,7 \%$ hipoactivo y $5,7 \%$ mixto.

El principal motivo de ingreso en los pacientes con delirium, fue infeccioso (51\%), seguido por causas metabólicas (16\%) y luego cardiovasculares (14\%); en cambio, en los pacientes sin delirium, la primera causa de hospitalización fue cardiovascular (45\%), seguida por causas infecciosas (24\%) y digestivas (22\%), estas diferencias fueron estadísticamente significativas al analizarlas con comparación de proporciones $(p<0,05)$.

Formulación de diagnóstico de delirium. La revisión de las fichas médicas de pacientes con delirium mostró que en 16,3\% no había descripción del examen mental del paciente al ingreso. Además, en 10,4\% adicional, se describe un examen mental «normal». La presencia de «elirium» o «Síndrome Confusional Agudo», como diagnóstico de ingreso se consignó sólo en 32,1\% de los pacientes, lo que diferenciando según el tipo de delirium ocurrió en $66,7 \%$ de los casos de delirium hiperactivos, el 23,5\% de los hipoactivos y ninguno de los mixtos.

Manejo de pacientes con delirium. El manejo no farmacológico de los pacientes ingresados con delirium no formó parte de las indicaciones del médico tratante. Hubo indicación de enfermería de utilizar medidas de contención física en 36\% de los casos de delirium, y analizando por tipo de delirium este método de manejo se usó en $58,3 \%$ de los delirium hiperactivo, 26,5\% de los hipoactivos y en $66,7 \%$ de los mixtos. Recibió kinesitera- pia motora $9,4 \%$ de los pacientes con delirium. No se aplicó protocolos de reorientación, iluminación diferenciada, respeto de horarios de sueño, como tampoco se utilizó medidas para evitar la inmovilización y la mayoría de las camas no contaba con barandas de protección.

El manejo farmacológico de pacientes con delirium se utilizó en $9,5 \%$ de los casos (7 pacientes); 4 pacientes recibieron exclusivamente haloperidol, 1 paciente haloperidol más trazodona, 2 pacientes combinaciones de haloperidol, clorpromazina y clorfenamina. La dosis inicial de haloperidol fue 2,5 ó $5 \mathrm{mg}$ en bolo endovenoso. La frecuencia en el uso de medicamentos fue diferente según el tipo de delirium, ocurriendo en $44,4 \%$ de los pacientes con delirium hiperactivo, en $7,4 \%$ de los hipoactivos y en ningún paciente con delirium mixto $(p<0,05)$.

Seguimiento de pacientes. Se realizó seguimiento intrahospitalario usando el instrumento CAM al $84,3 \%$ de los pacientes sin delirium al ingreso $(n=43)$, de éstos el 23,3\% (10 pacientes) desarrolló delirium durante la hospitalización.

Según la información de las epicrisis de los 108 pacientes, el promedio de estadía hospitalaria en el grupo con delirium fue de 10,21 días (DS 7,74), versus una estadía promedio de 5,78 días (DS 2,58 ) en los pacientes sin delirium al ingreso, diferencia estadísticamente significativa (test $\mathrm{T}$ Student, $\mathrm{p}<0,05)$. Fallecieron 4 pacientes del grupo con delirium (7\%) y ningún paciente en el grupo sin delirium.

\section{DisCUSIÓN}

El delirium es una condición muy frecuente entre los adultos mayores que son hospitalizados en el servicio de Medicina del Hospital de Urgencia de la Asistencia Pública. El 53\% de los pacientes AM ingresa con delirium y otro $23 \%$ lo desarrolla después de las primeras cuarenta y ocho horas de hospitalización, ubicándose en los rangos superiores de prevalencias descritas ${ }^{1,5-7}$ y mayor que lo descrito en pacientes de una unidad de cuidados intermedios chilena ${ }^{17}$. Esto podría explicarse en parte, porque el centro estudiado por nosotros recibe sólo pacientes hospitalizados de urgencia, no electivos y debido a la gran demanda asisten- 
cial ingresan sólo los más graves y por lo tanto con mayor riesgo de desarrollar delirium ${ }^{1,3}$.

A pesar de su prevalencia, es un problema severamente subdiagnosticado (68\% de los casos no se diagnostica, $16 \%$ de los pacientes deliriosos no tenía examen mental en el ingreso y $11 \%$ adicional estaba descrito como normal). El grado de subdiagnóstico se ubica en el rango superior de lo publicado en adultos mayores ${ }^{1,3,9}$, pero destaca el hecho que el síndrome confusional, en muchos casos (73\%) es descrito en el examen del paciente, pero no se agrega a los diagnósticos, dificultando así el enfrentamiento específico de esta patología 3,21 .

El tipo de delirium más frecuentemente encontrado fue el hipoactivo y también el menos diagnosticado. Esto es relevante, ya que hay datos que sugieren que ellos tendrían un pronóstico peor que los hiperactivos, sin haber claridad si esta diferencia es por el origen o el manejo de la patología 3 , ya que por tratarse de pacientes «ranquilos» no se pesquisa ni se trata este síndrome a tiempo.

Nuestros resultados concuerdan con la literatura, respecto a que los pacientes con delirium tienden a ser los más ancianos y aquellos más gravemente enfermos ${ }^{1-5}$, siendo el índice APACHE II mayor de 16 puntos el punto de corte elegido por Inouye 22 en su modelo predictivo de riesgo de desarrollar delirium en AM hospitalizados. También pudimos observar que los pacientes con patología infecciosa y trastornos metabólicos tienen significativamente mayor presencia de delirium.

Observamos que el manejo de este síndrome está lejos de cumplir con las recomendaciones internacionales y menos aún con las consideraciones especiales en el grupo de $\mathrm{AM}^{1,3,4,12}$. No se aplican medidas de prevención (iluminación diferenciada, respeto de ritmos de sueño-vigilia, reorientación repetitiva, ambiente tranquilo, etc.), diagnóstico precoz ni medidas de tratamiento específicas.

Este centro, que es un hospital público de urgencia, cuenta con limitados recursos persona- les y de infraestructura, dificultando el manejo de estos pacientes AM. Por ejemplo, no cuenta con medidas básicas de manejo ambiental, las salas de hospitalización están sometidas a un alto movimiento de pacientes, para prevenir caídas los pacientes deliriosos son inmovilizados con medidas de restricción física, que aumentan el riesgo del delirium y agitación en el AM ${ }^{4,11,23}$. El acceso de familiares como cuidadores está restringido y no se promueve que los pacientes permanezcan con sus elementos de apoyo sensorial como audífonos o lentes, lo que también favorece la aparición de delirium ${ }^{11,22}$.

En cuanto al manejo farmacológico, este centro cuenta con los medicamentos adecuados $3,4,12$, pero observamos que también se usan otros no recomendados, que pueden participar en la génesis del delirium (antihistamínicos) o tienen mayores efectos adversos (clorpromazina) en comparación con el haloperidol ${ }^{4}$. En cuanto a las dosis, cuando se usó haloperidol éste fue usado en dosis 2 a 5 veces mayores que las recomendadas para adultos mayores (2,5 ó $5 \mathrm{mg}$ iv en vez de iniciar con dosis bajas de 0,5 a $1 \mathrm{mg})^{12}$.

Se pudo corroborar que los pacientes con delirium durante su hospitalización, tuvieron una mayor estadía hospitalaria y mortalidad intrahospitalaria, como ha sido descrito en la literatura ${ }^{2,4}$.

Los resultados de este estudio, aun con las limitaciones propias de una investigación realizada en un solo centro y por un tiempo acotado, nos revelan un panorama inquietante que creemos no es, en ningún caso, exclusivo del centro asistencial evaluado, sino más bien una realidad mucho más amplia, que requiere mayor atención.

Este es el primer estudio chileno de incidencia de delirium en adultos mayores hospitalizados y los resultados nos muestran que es un síndrome severamente subdiagnosticado y subtratado. Esta realidad hace plantear la urgente necesidad de implementar programas de educación al equipo de salud en el diagnóstico precoz y manejo adecuado, especialmente dirigido a los AM. 


\section{REFERENCIAS}

1. González M, De Pablo J, Valdés M. Delirium: la confusión de los clínicos. Rev Méd Chile 2003; 131: 1051-60.

2. TRZepacz P. Delirium: Advances in diagnosis, pathophysiology and treatment. Psychiatr Clini North Am 1996; 19: 429-48.

3. MEAGHER D. Delirium: Optimizing management. BMJ 2001; 322: 144-50.

4. American Psychiatric Association. Practice guideline for the treatment of patients with delirium. Am J Psychiatry 1999; 156: 5 Supplement.

5. Cole MG. Delirium in elderly patients. Am J Geriatr Psychiatry 2004; 12: 7-21.

6. McCusker J, Cole M, Dendukuri N, Belzile E, Primeau F. Delirium in older medical inpatients and subsequent cognitive and functional status: a prospective study. CMAJ 2001; 165: 575-83.

7. BRITton A, Russeil R. Multidisciplinary team interventions for delirium in patients with chronic cognitive impairment. Cochrane Database of Systematic reviews 1, 2004.

8. LipowsKi Z. Delirium (acute confusional states). JAMA 1987; 258: 1789-92.

9. Francis J. Delirium in older patients. J Am Geriatr Soc 1992; 40: 829-38.

10. Rockwood K, Cosway S, Stolee P, Kydd D, Carver D, JARRET P. Increasing the recognition of delirium in elderly patients. J Am Geriatr Soc 1994; 42: 252-6.

11. Inouye $S$, Bogardus $S$, Charpentier P, Leo-Summers L, Acampora D, Holford T et al. A multicomponent intervention to prevent delirium in hospitalized older patients. N Engl J Med 1999; 340: 669-76.

12. Young L, George J. Guidelines for the diagnosis and management of delirium in the elderly. British Geriatric Society: www.bgs.org.uk/publications/publications.htm.

13. Marcantonio E, Flacker J, Wright J, Resnick N.
Reducing delirium after hip fracture: a randomized trial. JAGS 2001; 49: 516-22.

14. Flaherty J, Tariq S, Raghavan S, Bakshi S, MoINUDdin A, Morley J. A model for managing delirious older patients. JAGS 2003; 51: 1031-35.

15. OPS OMS. Perfil del Adulto mayor en Chile: desamollando respuestas integradas de sistemas de cuidado de salud para una población de rápido envejecimiento (INTRA). Publicación OPS/OMS 2002.

16. Hernández G, Ibáñez $C$, Kimelman M, Oreliana G, Montigo O, NúÑEZ C. Prevalencia de trastornos psiquiátricos en hombres y mujeres hospitalizados en un servicio de medicina interna de un hospital de Santiago de Chile. Rev Méd Chile 2001; 129: 1279-88.

17. González J, Barros J. Delirium en pacientes de una unidad de cuidados intermedios: estudio prospectivo. Rev Méd Chile 2000; 128: 75-9.

18. Inouye S, Van Dyck C, Alessi C, Balkin S, Siegal A, Horwitz R. Clarifying confusion: the confusion assessment method. Ann Intern Med 1990; 113: 941-48.

19. Knaus W, Draper E, Wagner D, Zimmerman J. APACHE II: a severity of disease classification system. Crit Care Med 1985; 13: 818-29.

20. McNicoll L, INouye S. Delirium. En Current Geriatric Diagnosis and Treatment. Lange Medical Books. Mac Graw-Hill 2004: 53-59.

21. Laurila JV, Pitkala KH, Strandberg T, Tilvis R. Detection and documentation of dementia and delirium in acute geriatric guards. Gen Hosp Psychiatry 2004; 26: 31-5.

22. Inouye $\mathrm{S}$, Viscoli $\mathrm{C}$, Horwitz R, Hurst L, Tinetti M. A predictive model for delirium in hospitalized elderly medical patients based on admission characteristics. Ann Intern Med 1993; 119: 474-81.

23. SuLvan-MarX E. Delirium and physical restraint in the hospitalized elderly. Image J Nurs Sch 1994; 26: 295-300. 